\title{
Limit cycles in planar piecewise linear Hamiltonian systems with three zones without equilibrium points
}

\author{
Alexander Fernandes da Fonseca* \\ Instituto de Matemática e Computação, Universidade Federal de Itajubá, \\ Avenida BPS 1303, Pinheirinho, CEP 37.500-903, Itajubá, MG, Brazil. \\ e-mail: alexff@unifei.edu.br \\ Jaume Llibre \\ Departament de Matemàtiques, Universitat Autònoma de Barcelona, \\ Bellaterra, 08193-Barcelona, Catalonia, Spain. \\ e-mail: jllibre@mat.uab.cat \\ Luis Fernando Mello \\ Instituto de Matemática e Computação, Universidade Federal de Itajubá, \\ Avenida BPS 1303, Pinheirinho, CEP 37.500-903, Itajubá, MG, Brazil. \\ e-mail: lfmelo@unifei.edu.br
}

\begin{abstract}
We study the existence of limit cycles in planar piecewise linear Hamiltonian systems with three zones without equilibrium points. In this scenario, we have shown that such systems have at most one crossing limit cycle.

Key-words: Piecewise smooth vector field, limit cycle, Hamiltonian vector field. 2010 Mathematics Subject Classification: Primary 34A36, 34C25, 34A26.

* Corresponding author.
\end{abstract}




\section{Introduction and statement of the main re- sults}

The study of piecewise smooth vector fields, especially the planar case, has grown over the last thirty years mainly due to its great applicability as a mathematical model of a series of applied phenomena in relay systems, mechanics, electrical circuits, among others. As a landmark for such studies, we cited the book of Andronov et al. [1], and more recently Filippov's book [5] and the book of di Bernardo et al. [2].

Many of the studies developed so far consider piecewise smooth vector fields with two zones and few studies are found with more zones. A piecewise smooth vector field with three zones in the plane is composed of three $C^{r}$ vector fields $X, Y$ and $Z, r \geq 1$, defined on $\mathbb{R}^{2}$ and separated by a pair of disjoint connected unbounded smooth curves $\Sigma_{1}$ and $\Sigma_{2}$. The separation curves $\Sigma_{i}$ are obtained by considering $\Sigma_{i}=h_{i}^{-1}(0)$, where $h_{i}: \mathbb{R}^{2} \longrightarrow \mathbb{R}$ are differentiable functions having 0 as a regular value, for $i=1,2$. Thus $\mathbb{R}^{2}=\mathcal{R}_{1} \cup \mathcal{R}_{2} \cup \mathcal{R}_{3} \cup \Sigma_{1} \cup \Sigma_{2}$, where the zones $\mathcal{R}_{i}, i=1,2,3$, are unbounded disjoint regions defined on the complement of the separation curves. So, a piecewise smooth vector field with three zones in the plane can be written as

$$
W(x, y)=\left\{\begin{array}{ll}
X(x, y), & h_{1}(x, y) \leq 0 \\
Y(x, y), & h_{1}(x, y) \geq 0 \\
Z(x, y), & h_{2}(x, y) \geq 0
\end{array} \text { and } \quad h_{2}(x, y) \leq 0,\right.
$$

System (1) will be denoted by $W=\left(X, Y, Z, \Sigma_{1}, \Sigma_{2}\right)$, or simply by $W=$ $(X, Y, Z)$ when the separation curves $\Sigma_{1}$ and $\Sigma_{2}$ are well understood.

We will use the vector field $X$ and the separation curve $\Sigma_{1}$ in the next definitions. However, they can be easily adapted to the vector fields $Y$ and $Z$ and the separation curve $\Sigma_{2}$.

The contact between the smooth vector field $X$ and the separation curve $\Sigma_{1}$ is characterized by the derivative of $h_{1}$ in the direction of the vector field $X$, that is, by the expression $X h_{1}(p)=\left\langle\nabla h_{1}(p), X(p)\right\rangle$, where $\langle\cdot, \cdot\rangle$ is the 
usual inner product in $\mathbb{R}^{2}$. Some important subsets of $\Sigma_{1}$ are the following ones (the same for $\Sigma_{2}$ ):

(a) Crossing set: $\Sigma_{1}^{c}=\left\{p \in \Sigma_{1} \mid X h_{1}(p) \cdot Y h_{1}(p)>0\right\}$;

(b) Sliding set: $\Sigma_{1}^{s}=\left\{p \in \Sigma_{1} \mid X h_{1}(p)<0, Y h_{1}(p)>0\right\}$;

(c) Escaping set: $\Sigma_{1}^{e}=\left\{p \in \Sigma_{1} \mid X h_{1}(p)>0, Y h_{1}(p)<0\right\}$.

When $p \in \Sigma_{1}$ and $X h_{1}(p)=0$ we say that $p$ is a tangential singularity of $X$. A tangential singularity is called a fold point of $X$ if $X h_{1}(p)=0$ but $X^{2} h_{1}(p) \neq 0$, where $X^{2} h_{1}(p)=\left\langle\nabla X h_{1}(p), X(p)\right\rangle$. Moreover, $p \in \Sigma_{1}$ is a visible (invisible, respectively) fold point of $X$ if $X h_{1}(p)=0$ and $X^{2} h_{1}(p)>0$ $\left(X^{2} h_{1}(p)<0\right.$, respectively).

In order to define a trajectory of a piecewise smooth vector field with three zones in the plane passing through a crossing point $p \in \Sigma_{1}$, it is enough to concatenate the trajectories of $X$ and $Y$ by $p$. However, in the sliding and escaping sets we need to define an auxiliary vector field and use the Filippov's convention (see [5]). We say that an equilibrium point $p$ of $X$ is real if $h_{1}(p)<0$ and it is virtual if $h_{1}(p)>0$.

A piecewise smooth vector field with three zones in the plane $W=$ $\left(X, Y, Z, \Sigma_{1}, \Sigma_{2}\right)$ is called continuous if

$$
X(p)=Y(p), \quad \forall p \in \Sigma_{1} \quad \text { and } \quad Y(q)=Z(q), \quad \forall q \in \Sigma_{2}
$$

Otherwise, it is called discontinuous.

Without being exhaustive we present some results relative to the study of piecewise smooth systems with three zones in the plane. A beautiful and simple example can be found in the introductory chapter of the book [4] where the authors studied the problem of a particle under the gravity law rolling in an inclined plane followed by a horizontal displacement and next moving in another inclined plane. This problem is modeled by a piecewise linear system with three zones in the plane with a continuous of equilibria. 
In fact, as far as we know, the studies found in the pertinent literature deal with piecewise smooth systems with three zones in the plane with at least one equilibrium point which can be real or virtual. This is the case, for instance, in the studies carried out in [3], [6], [7], [8], [9], [10]. We emphasize that in these articles the authors studied the existence and the number of limit cycles under the hypothesis of the continuity of the vector fields in the separation curves which are straight lines.

In this article we are mainly interested in the study of the existence and the number of crossing limit cycles of a piecewise smooth vector field with three zones in the plane $W=\left(X, Y, Z, \Sigma_{1}, \Sigma_{2}\right)$ under the following assumptions:

H1. The separation curves $\Sigma_{1}$ and $\Sigma_{2}$ are straight lines.

H2. The vector fields $X, Y$ and $Z$ are linear.

H3. The vector fields $X, Y$ and $Z$ are Hamiltonian.

H4. The vector fields $X, Y$ and $Z$ have no equilibrium points, neither real nor virtual.

We emphasize that when the vector fields $X, Y$ and $Z$ are Hamiltonian the solution curves of the respective differential equations are contained in the level sets of the Hamiltonian functions. This greatly simplifies the analysis of the transitions maps in and between the separation curves by seeking for points in $\Sigma_{i}$ that are on the same level curves of these Hamiltonian functions. In this case, we avoid working with transition times.

We can state the main results of this article.

Theorem 1. Continuous planar piecewise linear Hamiltonian systems with three zones separated by two straight lines and without equilibrium points, neither real nor virtual, have no crossing limit cycles. 
Theorem 2. Discontinuous planar piecewise linear Hamiltonian systems with three zones separated by two straight lines and without equilibrium points, neither real nor virtual, have at most one crossing limit cycle.

The article is organized as follows. In section 2 are presented the proofs of Theorems 1 and 2. Examples of discontinuous planar piecewise linear Hamiltonian systems with three zones separated by two straight lines and without equilibrium points, neither real nor virtual, are analyzed in section 3. In particular, we give an example of a discontinuous planar piecewise linear Hamiltonian system with three zones separated by two straight lines and without equilibrium points, neither real nor virtual, with one crossing limit cycle.

\section{Proofs of Theorems 1 and 2}

Without loss of generality, from the assumption $\mathbf{H 1}$, we can take $h_{1}, h_{2}$ : $\mathbb{R}^{2} \longrightarrow \mathbb{R}$ given by $h_{1}(x, y)=x+1$ and $h_{2}(x, y)=x-1$. Thus, the separation lines can be written as

$$
\begin{aligned}
& \Sigma_{1}=h_{1}^{-1}(0)=\left\{(x, y) \in \mathbb{R}^{2}: x=-1\right\} \\
& \Sigma_{2}=h_{2}^{-1}(0)=\left\{(x, y) \in \mathbb{R}^{2}: x=1\right\}
\end{aligned}
$$

These two straight lines decompose the plane into three regions: $\mathcal{R}_{L}=$ $\left\{(x, y) \in \mathbb{R}^{2}: x<-1\right\}, \mathcal{R}_{C}=\left\{(x, y) \in \mathbb{R}^{2}:-1<x<1\right\}, \mathcal{R}_{R}=\{(x, y) \in$ $\left.\mathbb{R}^{2}: x>1\right\}$. Thus, $\mathbb{R}^{2}=\mathcal{R}_{L} \cup \mathcal{R}_{C} \cup \mathcal{R}_{R} \cup \Sigma_{1} \cup \Sigma_{2}$.

Taking into account the assumptions $\mathbf{H 2}, \mathbf{H 3}$ and $\mathbf{H 4}$, we can write a piecewise linear Hamiltonian vector field with three zones and without equilibrium points in the plane as

$$
W(x, y)= \begin{cases}\mathcal{X}_{L}(x, y), & x \leq-1 \\ \mathcal{X}_{C}(x, y), & -1 \leq x \leq 1 \\ \mathcal{X}_{R}(x, y), & x \geq 1\end{cases}
$$


where

$$
\begin{aligned}
& \mathcal{X}_{L}(x, y)=\left(-\lambda_{L} b_{L} x+b_{L} y+\gamma_{L},-\lambda_{L}^{2} b_{L} x+\lambda_{L} b_{L} y+\delta_{L}\right), \\
& \mathcal{X}_{C}(x, y)=\left(-\lambda_{C} b_{C} x+b_{C} y+\gamma_{C},-\lambda_{C}^{2} b_{C} x+\lambda_{C} b_{C} y+\delta_{C}\right), \\
& \mathcal{X}_{R}(x, y)=\left(-\lambda_{R} b_{R} x+b_{R} y+\gamma_{R},-\lambda_{R}^{2} b_{R} x+\lambda_{R} b_{R} y+\delta_{R}\right),
\end{aligned}
$$

and $\delta_{k} \neq \lambda_{k} \gamma_{k}, k \in\{L, C, R\}$. In fact, in order to satisfy conditions $\mathbf{H 2}$ and H4 these vector fields can be written as

$$
\mathcal{X}_{k}(x, y)=\left(a_{k} x+b_{k} y+\gamma_{k}, \lambda_{k} a_{k} x+\lambda_{k} b_{k} y+\delta_{k}\right),
$$

where $\delta_{k} \neq \lambda_{k} \gamma_{k}, k \in\{L, C, R\}$. Then in order to satisfy condition $\mathbf{H 3}$ the divergence of (3) must be zero, that is $a_{k}=-\lambda_{k} b_{k}$.

If $b_{k}=0$ for some $k \in\{L, C, R\}$, then the vector field $\mathcal{X}_{k}$ has the form $\mathcal{X}_{k}(x, y)=\left(\gamma_{k}, \delta_{k}\right)$, with $\delta_{k} \neq \lambda_{k} \gamma_{k}$. So the trajectories are straight lines implying that $W$ has no periodic orbits. Henceforth, we consider $b_{k} \neq 0$, for $k \in\{L, C, R\}$.

As the vector fields $\mathcal{X}_{L}, \mathcal{X}_{C}$ and $\mathcal{X}_{R}$ in (2) are linear and Hamiltonian, there are quadratic functions $\mathcal{H}_{k}: \mathbb{R}^{2} \longrightarrow \mathbb{R}$ (Hamiltonian functions) such that

$$
\mathcal{X}_{k}(x, y)=\left(-\frac{\partial \mathcal{H}_{k}}{\partial y}(x, y), \frac{\partial \mathcal{H}_{k}}{\partial x}(x, y)\right), \quad k \in\{L, C, R\} .
$$

By elementary calculations, we found

$$
\mathcal{H}_{k}(x, y)=-\frac{\lambda_{k}^{2} b_{k}}{2} x^{2}+\lambda_{k} b_{k} x y-\frac{b_{k}}{2} y^{2}+\delta_{k} x-\gamma_{k} y, \quad k \in\{L, C, R\} .
$$

From the assumption of the continuity of $W$ expressed in Theorem 1, we obtain

$$
\mathcal{X}_{L}(-1, y)=\mathcal{X}_{C}(-1, y) \quad \text { and } \quad \mathcal{X}_{C}(1, y)=\mathcal{X}_{R}(1, y), \quad \forall y \in \mathbb{R} .
$$

These equalities imply that

$$
\begin{array}{ll}
\lambda_{L}=\lambda_{C}=\lambda_{R}=\lambda, & b_{L}=b_{C}=b_{R}=b, \\
\gamma_{L}=\gamma_{C}=\gamma_{R}=\gamma, & \delta_{L}=\delta_{C}=\delta_{R}=\delta .
\end{array}
$$


Therefore, from (2), a continuous piecewise linear Hamiltonian vector field with three zones and without equilibrium points in the plane can be written as

$$
W(x, y)=\left(-\lambda b x+b y+\gamma,-\lambda^{2} b x+\lambda b y+\delta\right), \quad \delta \neq \lambda \gamma, \quad b \neq 0 .
$$

By hypothesis this vector field has no equilibrium points. So it has no periodic orbits and, in particular, it has no limit cycles. In short, Theorem 1 is proved.

Now we will begin the proof of Theorem 2 .

From (2) a piecewise linear Hamiltonian vector field with three zones and without equilibrium points in the plane has a crossing periodic orbit if there are crossing points $\left(-1, y_{0}\right),\left(-1, y_{3}\right) \in \Sigma_{1}$ and crossing points $\left(1, y_{1}\right),\left(1, y_{2}\right) \in$ $\Sigma_{2}$ such that

$$
\begin{aligned}
\mathcal{H}_{L}\left(-1, y_{0}\right) & =\mathcal{H}_{L}\left(-1, y_{3}\right), \\
\mathcal{H}_{C}\left(-1, y_{0}\right) & =\mathcal{H}_{C}\left(1, y_{1}\right), \\
\mathcal{H}_{R}\left(1, y_{1}\right) & =\mathcal{H}_{R}\left(1, y_{2}\right), \\
\mathcal{H}_{C}\left(1, y_{2}\right) & =\mathcal{H}_{C}\left(-1, y_{3}\right) .
\end{aligned}
$$

In fact, in this situation, if we take

$$
\begin{aligned}
\alpha_{L} & =\mathcal{H}_{L}^{-1}\left(\left\{\mathcal{H}_{L}\left(-1, y_{0}\right)\right\}\right) \cap \overline{\mathcal{R}_{L}}, \\
\alpha_{C, 1} & =\mathcal{H}_{C}^{-1}\left(\left\{\mathcal{H}_{C}\left(-1, y_{0}\right)\right\}\right) \cap \overline{\mathcal{R}_{C}}, \\
\alpha_{C, 2} & =\mathcal{H}_{C}^{-1}\left(\left\{\mathcal{H}_{C}\left(-1, y_{3}\right)\right\}\right) \cap \overline{\mathcal{R}_{C}}, \\
\alpha_{R} & =\mathcal{H}_{R}^{-1}\left(\left\{\mathcal{H}_{R}\left(1, y_{1}\right)\right\}\right) \cap \overline{\mathcal{R}_{R}},
\end{aligned}
$$

then

$$
\alpha=\alpha_{R} \cup \alpha_{C, 1} \cup \alpha_{L} \cup \alpha_{C, 2}
$$

is a crossing periodic orbit of $W$. See Figure 1. 


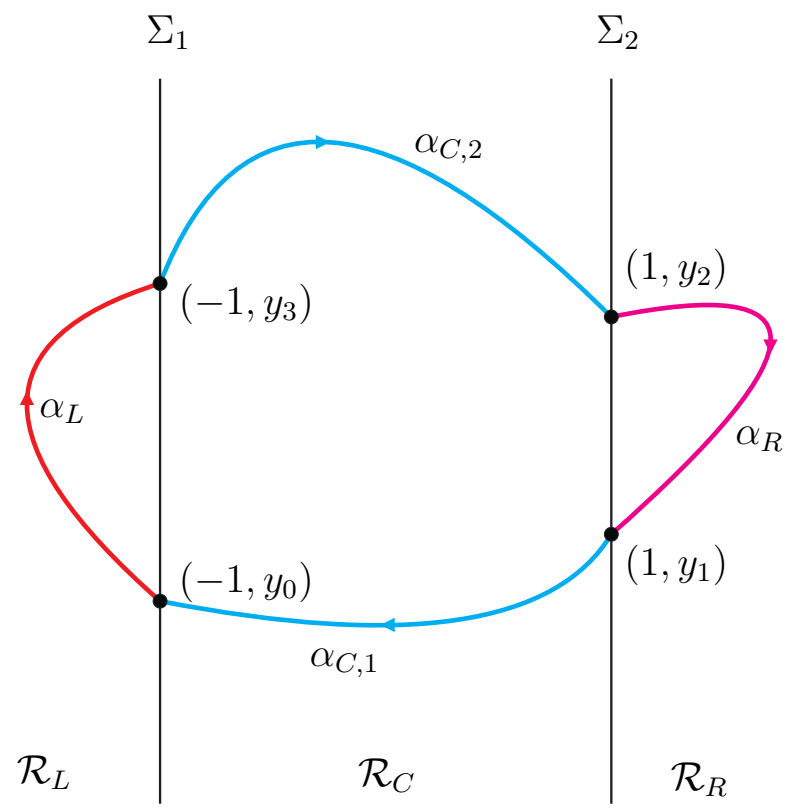

Figure 1: A crossing periodic orbit of $W: \alpha=\alpha_{R} \cup \alpha_{C, 1} \cup \alpha_{L} \cup \alpha_{C, 2}$.

From the above four equations in (6) and (4), we obtain

$$
\begin{aligned}
-\frac{1}{2}\left(y_{0}-y_{3}\right)\left(2 b_{L} \lambda_{L}+b_{L} y_{0}+b_{L} y_{3}+2 \gamma_{L}\right) & =0, \\
-\lambda_{C} b_{C} y_{0}-\frac{1}{2} b_{C} y_{0}{ }^{2}-2 \delta_{C}-\gamma_{C} y_{0}-\lambda_{C} b_{C} y_{1}+\frac{1}{2} b_{C} y_{1}{ }^{2}+\gamma_{C} y_{1} & =0, \\
-\frac{1}{2}\left(y_{1}-y_{2}\right)\left(-2 b_{R} \lambda_{R}+b_{R} y_{1}+b_{R} y_{2}+2 \gamma_{R}\right) & =0, \\
\lambda_{C} b_{C} y_{2}-\frac{1}{2} b_{C} y_{2}{ }^{2}+2 \delta_{C}-\gamma_{C} y_{2}+\lambda_{C} b_{C} y_{3}+\frac{1}{2} b_{C} y_{3}{ }^{2}+\gamma_{C} y_{3} & =0 .
\end{aligned}
$$

As $y_{0} \neq y_{3}$ and $y_{1} \neq y_{2}$, equation (7) can be solved for $y_{0}$ as well as equation (9) can be solved for $y_{2}$. Substituting the obtained expressions of $y_{0}$ and $y_{2}$ into equations (8) and (10), respectively, we get the equations of two hyperbolas in the plane $y_{1} y_{3}$ given by

$$
\frac{\left(y_{1}-A\right)^{2}}{K}-\frac{\left(y_{3}-B\right)^{2}}{K}=1, \quad \frac{\left(y_{1}-C\right)^{2}}{K}-\frac{\left(y_{3}-D\right)^{2}}{K}=1,
$$


where

$$
\begin{aligned}
& A=\lambda_{C}-\frac{\gamma_{C}}{b_{C}}, \\
& B=-2 \frac{\gamma_{L}}{b_{L}}-2 \lambda_{L}+\lambda_{C}+\frac{\gamma_{C}}{b_{C}}, \\
& C=-2 \frac{\gamma_{R}}{b_{R}}+2 \lambda_{R}-\lambda_{C}+\frac{\gamma_{C}}{b_{C}}, \\
& D=-\left(\lambda_{C}+\frac{\gamma_{C}}{b_{C}}\right), \\
& K=4 \frac{\delta_{C}-\lambda_{C} \gamma_{C}}{b_{C}} .
\end{aligned}
$$

We have the following three possibilities:

Case 1. The hyperbolas do not intersect. So, the discontinuous piecewise linear Hamiltonian vector field with three zones and without equilibrium points in the plane given in equation (2) has no periodic solutions. See Example 1 .

Case 2. The hyperbolas coincide. In this case, the discontinuous piecewise linear Hamiltonian vector field with three zones and without equilibrium points in the plane given in equation (2) has infinitely many periodic solutions. See Example 2.

Case 3. The hyperbolas intersect at two points. Thus, the discontinuous piecewise linear Hamiltonian vector field with three zones and without equilibrium points in the plane given in equation (2) has only one limit cycle. It is easy to check that the two hyperbolas (11) at most can intersect at two points. See Example 3.

In short, under the hypotheses of Theorem 2, a discontinuous piecewise linear Hamiltonian vector field with three zones and without equilibrium points in the plane has at most one limit cycle. Theorem 2 is proved. 


\section{Some examples}

In Figures 2, 3 and 4, related to Examples 1, 2, 3, we will use the following notations: the separation lines $\Sigma_{1}$ and $\Sigma_{2}$ are illustrated in dashed lines while the sliding sets are depicted in black solid lines. Black dots are used for fold points.

Example 1. Consider $W=\left(\mathcal{X}_{L}, \mathcal{X}_{C}, \mathcal{X}_{R}\right)$ defined by

$$
\begin{aligned}
& \mathcal{X}_{L}(x, y)=(-x+y+1,-x+y+3), \\
& \mathcal{X}_{C}(x, y)=(-x+y+1,-x+y+2), \\
& \mathcal{X}_{R}(x, y)=(-2 x+y+1,-4 x+2 y+1) .
\end{aligned}
$$

In this case we have

$$
\begin{array}{llll}
b_{L}=1, & \lambda_{L}=1, & \gamma_{L}=1, & \delta_{L}=3, \\
b_{C}=1, & \lambda_{C}=1, & \gamma_{C}=1, & \delta_{C}=2, \\
b_{R}=1, & \lambda_{R}=2, & \gamma_{R}=1, & \delta_{R}=1 .
\end{array}
$$

The Hamiltonian functions (4) are, respectively, given by

$$
\begin{aligned}
& \mathcal{H}_{L}(x, y)=-\frac{x^{2}}{2}+x y-\frac{y^{2}}{2}+3 x-y, \\
& \mathcal{H}_{C}(x, y)=-\frac{x^{2}}{2}+x y-\frac{y^{2}}{2}+2 x-y \\
& \mathcal{H}_{R}(x, y)=-2 x^{2}+2 x y-\frac{y^{2}}{2}+x-y .
\end{aligned}
$$

Then, the equations of the two hyperbolas (11) are

$$
\frac{y_{1}^{2}}{4}-\frac{\left(y_{3}+2\right)^{2}}{4}=1, \quad \frac{\left(y_{1}-2\right)^{2}}{4}-\frac{\left(y_{3}+2\right)^{2}}{4}=1,
$$

It is easy to show that the two hyperbolas do not intersect.

The point $(-1,-2) \in \Sigma_{1}$ is an invisible fold point of $\mathcal{X}_{L}$ and a visible fold point of $\mathcal{X}_{C}$. The point $(1,0) \in \Sigma_{2}$ is an invisible fold point of $\mathcal{X}_{C}$ while the point $(1,1) \in \Sigma_{2}$ is an invisible fold point of $\mathcal{X}_{R}$. They are the endpoints of the sliding set in $\Sigma_{2}$. The phase portrait of $W$ is depicted in Figure 2. 


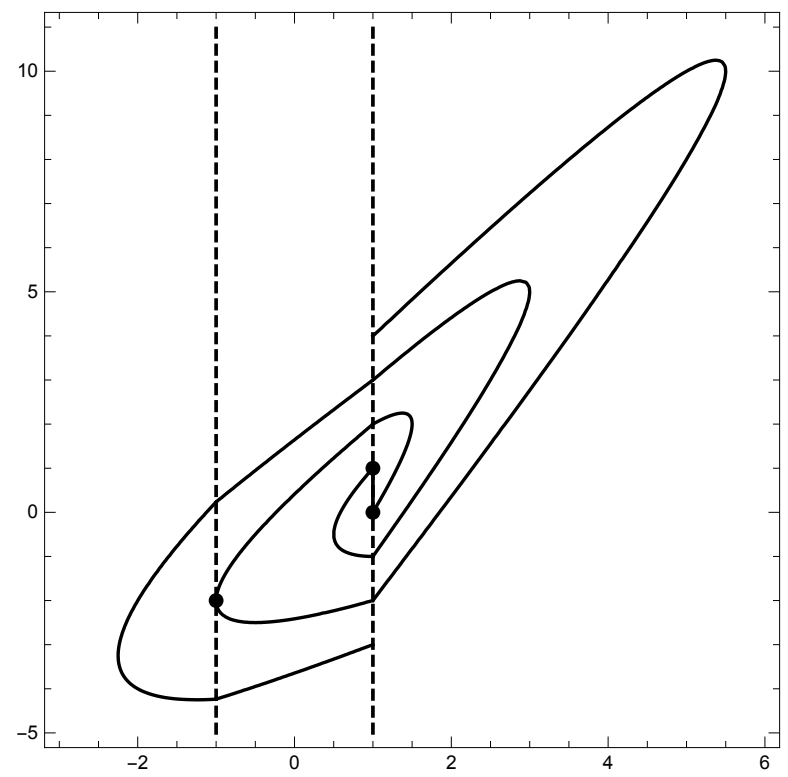

Figure 2: Phase portrait of $W$ defined by (12), (13) and (14).

Example 2. Considering the parameters

$$
\begin{array}{llll}
b_{L}=1, & \lambda_{L}=1, & \gamma_{L}=1, & \delta_{L}=3, \\
b_{C}=1, & \lambda_{C}=1, & \gamma_{C}=1, & \delta_{C}=2, \\
b_{R}=1, & \lambda_{R}=1, & \gamma_{R}=1, & \delta_{R}=-1,
\end{array}
$$

$W=\left(\mathcal{X}_{L}, \mathcal{X}_{C}, \mathcal{X}_{R}\right)$ can be written as

$$
\begin{aligned}
& \mathcal{X}_{L}(x, y)=(-x+y+1,-x+y+3), \\
& \mathcal{X}_{C}(x, y)=(-x+y+1,-x+y+2), \\
& \mathcal{X}_{R}(x, y)=(-x+y+1,-x+y-1) .
\end{aligned}
$$




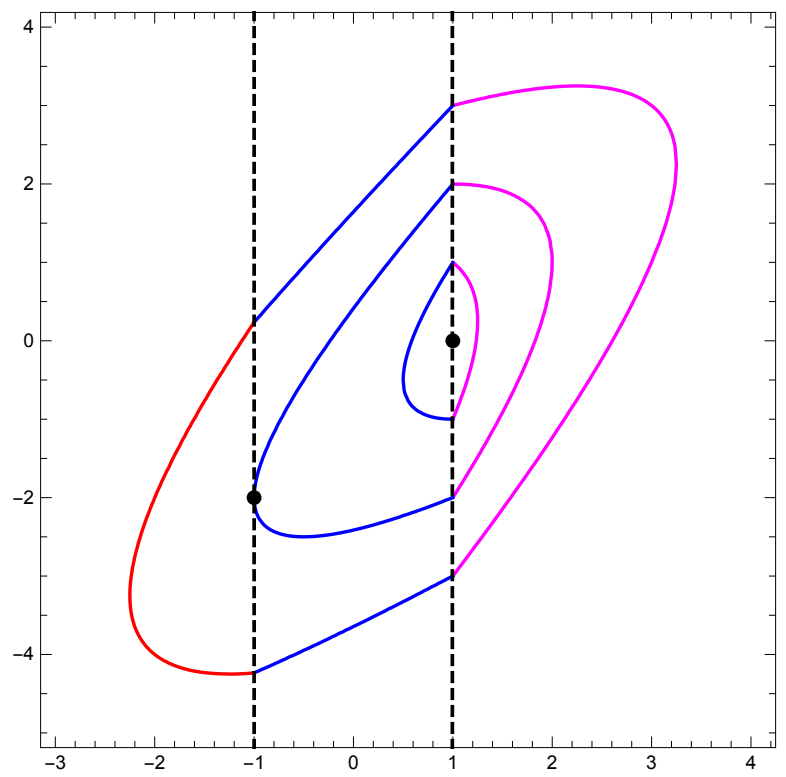

Figure 3: Phase portrait of $W$ defined by (15), (16) and (17).

So the Hamiltonian functions (4) have the expressions

$$
\begin{aligned}
& \mathcal{H}_{L}(x, y)=-\frac{x^{2}}{2}+x y-\frac{y^{2}}{2}+3 x-y, \\
& \mathcal{H}_{C}(x, y)=-\frac{x^{2}}{2}+x y-\frac{y^{2}}{2}+2 x-y, \\
& \mathcal{H}_{R}(x, y)=-\frac{x^{2}}{2}+x y-\frac{y^{2}}{2}-x-y .
\end{aligned}
$$

The equations of the two hyperbolas (11) are equal and given by

$$
\frac{y_{1}^{2}}{4}-\frac{\left(y_{3}+2\right)^{2}}{4}=1
$$

The point $(-1,-2) \in \Sigma_{1}$ is an invisible fold point of $\mathcal{X}_{L}$ and a visible fold point of $\mathcal{X}_{C}$. The point $(1,0) \in \Sigma_{2}$ is an invisible fold point of both $\mathcal{X}_{C}$ and $\mathcal{X}_{R}$. The phase portrait of $W$ is illustrated in Figure 3. 
Example 3. From equation (2), consider $W=\left(\mathcal{X}_{L}, \mathcal{X}_{C}, \mathcal{X}_{R}\right)$ defined by

$$
\begin{aligned}
& \mathcal{X}_{L}(x, y)=(-x+y+1,-x+y+3), \\
& \mathcal{X}_{C}(x, y)=(-x+y-1,-x+y+2), \\
& \mathcal{X}_{R}(x, y)=(-2 x+y+1,-4 x+2 y-2) .
\end{aligned}
$$

In this case we have

$$
\begin{array}{llll}
b_{L}=1, & \lambda_{L}=1, & \gamma_{L}=1, & \delta_{L}=3, \\
b_{C}=1, & \lambda_{C}=1, & \gamma_{C}=-1, & \delta_{C}=2, \\
b_{R}=1, & \lambda_{R}=2, & \gamma_{R}=1, & \delta_{R}=-2 .
\end{array}
$$

Then the Hamiltonian functions (4) are, respectively, given by

$$
\begin{aligned}
& \mathcal{H}_{L}(x, y)=-\frac{x^{2}}{2}+x y-\frac{y^{2}}{2}+3 x-y, \\
& \mathcal{H}_{C}(x, y)=-\frac{x^{2}}{2}+x y-\frac{y^{2}}{2}+2 x+y, \\
& \mathcal{H}_{R}(x, y)=-2 x^{2}+2 x y-\frac{y^{2}}{2}-2 x-y .
\end{aligned}
$$

Equations (7), (8), (9) and (10) can be written as

$$
\begin{aligned}
\frac{1}{2}\left(y_{0}-y_{3}\right)\left(y_{0}+y_{3}+4\right) & =0, \\
\frac{1}{2}\left(-y_{0}^{2}+y_{1}^{2}-4 y_{1}-8\right) & =0, \\
-\frac{1}{2}\left(y_{1}-y_{2}\right)\left(y_{1}+y_{2}-2\right) & =0, \\
\frac{1}{2}\left(-y_{2}^{2}+4 y_{2}+y_{3}^{2}+8\right) & =0,
\end{aligned}
$$

which imply that the equations of the two hyperbolas (11) have the form

$$
\frac{\left(y_{1}-2\right)^{2}}{12}-\frac{\left(y_{3}+4\right)^{2}}{12}=1, \quad \frac{y_{1}^{2}}{12}-\frac{y_{3}^{2}}{12}=1 .
$$

As $y_{0} \neq y_{3}$ and $y_{1} \neq y_{2}$, the real symmetric solutions of the above system are

$$
y_{0}=-2-\sqrt{5}, \quad y_{1}=1-2 \sqrt{5}, \quad y_{2}=1+2 \sqrt{5}, \quad y_{3}=-2+\sqrt{5},
$$




$$
y_{0}=-2+\sqrt{5}, \quad y_{1}=1+2 \sqrt{5}, \quad y_{2}=1-2 \sqrt{5}, \quad y_{3}=-2-\sqrt{5} .
$$

Note that both identities provide the same four points on the separation lines $\Sigma_{1}$ and $\Sigma_{2}$. The points $\left(-1, y_{0}\right),\left(-1, y_{3}\right) \in \Sigma_{1}$, and $\left(1, y_{1}\right),\left(1, y_{2}\right) \in \Sigma_{2}$ are crossing points because

$$
\begin{aligned}
\left(\mathcal{X}_{L}\left(-1, y_{0}\right) \cdot(1,0)\right)\left(\mathcal{X}_{C}\left(-1, y_{0}\right) \cdot(1,0)\right) & =2 \sqrt{5}+5>0 \\
\left(\mathcal{X}_{L}\left(-1, y_{3}\right) \cdot(1,0)\right)\left(\mathcal{X}_{C}\left(-1, y_{3}\right) \cdot(1,0)\right) & =5-2 \sqrt{5}>0 \\
\left(\mathcal{X}_{C}\left(1, y_{1}\right) \cdot(1,0)\right)\left(\mathcal{X}_{R}\left(1, y_{1}\right) \cdot(1,0)\right) & =2(\sqrt{5}+10)>0 \\
\left(\mathcal{X}_{C}\left(1, y_{2}\right) \cdot(1,0)\right)\left(\mathcal{X}_{R}\left(1, y_{2}\right) \cdot(1,0)\right) & =20-2 \sqrt{5}>0
\end{aligned}
$$

So the level sets of $\mathcal{H}_{k}$ containing the points

$$
(-1,-2-\sqrt{5}),(-1,-2+\sqrt{5}) \in \Sigma_{1} \quad \text { and } \quad(1,1-2 \sqrt{5}),(1,1+2 \sqrt{5}) \in \Sigma_{2}
$$

form a crossing limit cycle of $W=\left(\mathcal{X}_{L}, \mathcal{X}_{C}, \mathcal{X}_{R}\right)$ defined by (18), (19) and (20), see Figure 4. This crossing limit cycle is attractor since the associated Poincaré map can be written as

$$
P\left(y_{0}\right)=-\sqrt{y_{0}^{2}+4 \sqrt{y_{0}^{2}+8 y_{0}+28}+8 y_{0}+20},
$$

and its derivative evaluated at $y_{0}=-2-\sqrt{5}$ gives

$$
P^{\prime}(-2-\sqrt{5})=\frac{1}{19}(109-48 \sqrt{5})
$$

which satisfies $0<P^{\prime}(-2-\sqrt{5})<1$.

The point $(-1,-2) \in \Sigma_{1}$ is an invisible fold point of $\mathcal{X}_{L}$ and the point $(-1,0) \in \Sigma_{1}$ is a visible fold point of $\mathcal{X}_{C}$. They are the endpoints of the sliding set in $\Sigma_{1}$. The point $(1,1) \in \Sigma_{2}$ is an invisible fold point of $\mathcal{X}_{R}$ while the point $(1,2) \in \Sigma_{2}$ is an invisible fold point of $\mathcal{X}_{C}$, and they are the endpoints of the sliding set in $\Sigma_{2}$. 


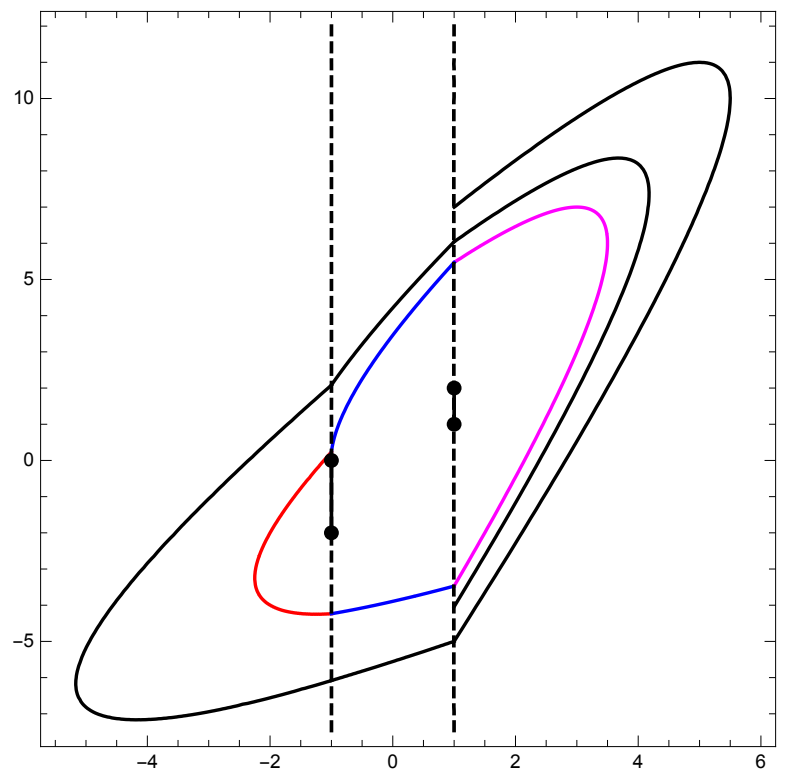

Figure 4: Phase portrait of $W$ defined by (18), (19) and (20) with the attracting crossing limit cycle.

\section{Conflict of interest}

The authors declare that they have no conflict of interest.

Acknowledgements. The first and third authors are partially supported by Fundação de Amparo à Pesquisa do Estado de Minas Gerais - FAPEMIG [grant number APQ-01158-17]. The second author is partially supported by the Ministerio de Ciencia, Innovación y Universidades, Agencia Estatal de Investigación grants MTM2016-77278-P (FEDER), the Agència de Gestió d'Ajuts Universitaris i de Recerca grant 2017SGR1617, and the H2020 European Research Council grant MSCA-RISE-2017-777911.

\section{References}

[1] A. Andronov, A. Vitt, S. Khaikin, Theory of Oscillators, Pergamon Press Inc., UK, 1966. 
[2] M. di Bernardo, C.J. Budd, A.R. Champneys, P. KowalCZYK, Piecewise-Smooth Dynamical Systems: Theory and Applications, Springer-Verlag, London, 2008.

[3] R. Euzébio, R. PAzim, E. Ponce, Jump bifurcations in some degenerate planar piecewise linear differential systems with three zones, Phys. D, 325 (2016), 74-85.

[4] M. FeČKan, M. PospíšIl, Poincaré-Andronov-Melnikov Analysis for Non-Smooth Systems. Elsevier/Academic Press, Amsterdam, 2016.

[5] A.F. Filippov, Differential Equations With Discontinuous Righthand Sides, Kluwer Academic Publishers Group, Dordrecht, 1988.

[6] E. Freire, E. Ponce, F. Rodrigo, F. Torres, Bifurcation sets of symmetrical continuous piecewise linear systems with three zones, Internat. J. Bifur. Chaos Appl. Sci. Engrg., 12 (2002), 1675-1702.

[7] S. Li, J. LliBRE, Phase portraits of continuous piecewise linear Liénard differential systems with three zones, Chaos Solitons Fractals, 120 (2019), 149-157.

[8] M.F.S. Lima, J. LliBRE, Limit cycles for a class of continuous piecewise linear differential systems with three zones, Internat. J. Bifur. Chaos Appl. Sci. Engrg., 22 (2012), 1250138, 10 pp.

[9] M.F.S. Lima, C. Pessoa, W.F. Pereira, Limit cycles bifurcating from a period annulus in continuous piecewise linear differential systems with three zones, Internat. J. Bifur. Chaos Appl. Sci. Engrg., 27 (2017), 1750022, 14 pp.

[10] J. Llibre, E. Ponce, C. VAlls, Uniqueness and non-uniqueness of limit cycles for piecewise linear differential systems with three zones and no symmetry, J. Nonlinear Sci., 25 (2015), 861-887. 